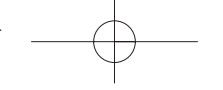

MARKETING

PAR PATRICK AUDEBERT-LASROCHAS

\title{
Comment différencier négociation et vente
}

La vente et la négociation sont restées très longtemps des concepts différenciés; vendre était directement lié à une transaction commerciale alors que la négociation restait I'apanage, soit des diplomates, soit des managers pour les relations sociales. Depuis plusieurs années nous constatons, dans la pratique des affaires, une confusion des deux fonctions.
$\mathrm{L}$

a perception et/ou l'utilisation des concepts de « vente » et « négociation » ont changé ces dernières décennies. Pendant longtemps la vente est restée exclusivement liée à l'acte commercial entre partenaires de type acheteur-vendeur; les mots et expressions: techniques de vente, équipes de vente, chef des ventes, vente à l'arraché, vente sur place ou plus récemment vente directe, sont couramment utilisés dans l'entreprise.

Quant à la notion de négociation, elle est restée très longtemps l'apanage des diplomates pour régler les relations internationales et des managers pour gérer les relations sociales au sein des entreprises et des organisations.

Depuis quelques années, la notion de vente va bien audelà de l'acte commercial; on « vend » ses idées. À titre d'exemple, à propos de la guerre en Irak: « Derrière ces maîtres de guerre, une armée de gourous se charge de vendre le conflit ${ }^{1} \gg$.

La notion de négociation a marqué une double évolution:

1. l'emploi courant de l'expression « négociation commerciale » dans le langage managérial; dans la plupart

1. VSD, 27 mars 2003 ; La Voix du Nord, 19 octobre 2002 ; Le Figaro, 14 janvier 2003. 
des écoles de commerce, mais également dans les formations universitaires à la gestion, le cours de techniques de vente a été remplacé par le cours de négociation commerciale ;

2. le concept négociation est de plus en plus galvaudé et utilisé dans des situations qui n'ont rien à voir avec la négociation; par exemple: au sujet d'un match de volleyball, ce titre: «Bien négocier ce rendezvous important $»^{2}$ ou ce commentaire: « Écarté du siège de leader, Marseille accueille le Stade rennais pour une rencontre délicate à négocier $»^{3}$.

Ces constatations induisent les questions suivantes: Négocie-t-on lorsque l'on vend? Peut-on négocier sans vendre ou vendre sans négocier? Quelles sont les spécificités et les limites de ces deux notions? La suite de cet article essayera d'apporter des réponses cohérentes à ces interrogations. Pour ce faire, cet article est composé de deux grandes parties:

- L'état des lieux, qui partira de l'hypothèse suivante: «Il y a confusion dans la perception de la différence entre le concept de vente et celui de la négociation. ».

- À partir de cette hypothèse, nous soumettrons cinq propositions pour tenter de clarifier ces deux concepts.

\section{I. - L'ÉTAT DES LIEUX}

Quel que soit l'angle «d'attaque » de ce problème, à savoir l'identification des spécifités de la vente et de la négociation, autrement dit, les différences et les points communs entre ces deux notions, on retombe sur un «certain flou » pour ne pas dire un sentiment de confusion.

Pour illustrer ce constat, nous allons balayer cinq grandes sources d'information, à savoir: la presse, les organismes de formation, les interviews, les enquêtes, la littérature spécialisée.

\section{La Presse}

Nous avons, lors de l'introduction, cité plusieurs cas que nous pouvons compléter par les exemples suivants: "La vente est une comédie humaine qui doit apprendre à se sortir de situations difficiles. ${ }^{4}$ » Dans un autre domaine, le maire de la ville de Béthune (Pas de Calais) a décidé de voter l'implantation d'une grande surface commerciale; le député local, adversaire politique du maire, affirme: «Un marchandage politicien a été organisé par le maire qui a négocié son avis favorable à hauteur de 5,3 millions d'euros... tout est-il à vendre? » se demande le député ${ }^{5}$. Où, en plus de la notion de négociation et vente, vient se greffer le concept de marchandage. Pour en revenir à la fonction commerciale, une journaliste, Anne-Françoise Rabaud ${ }^{6}$, donne la parole à Fabien Lezeau de la société FLZ conseil; dans cet article, ce consultant y présente une démarche originale. Il met ensemble, dans le même séminaire, acheteurs et vendeurs; Fabien Lezeau affirme: "Chacun agit en fonction de la représentation qu'il a de l'autre. Acheteurs et vendeurs vont pouvoir trouver, $[\ldots]$ de nouvelles façons de faire, de nouvelles

\footnotetext{
2. La Voix du Nord, 19 octobre 2002.

3. Le Figaro, 14 janvier 2003.

4. G.-H. Bourgeois, in Action Commerciale, $\mathrm{n}^{\circ} 193$.

5. Voix du Nord, 16 mai 2003.

6. Action Commerciale, $\mathrm{n}^{\circ} 202$.
} 
options de travail pour la négociation. » Dans le même article, la journaliste interviewe Guillaume Huot, responsable des formations marketing dans un grand organisme de formation, qui déclare: « Le cœur de l'activité de formation reste ancré sur les fondamentaux - l'écoute, la reformulation, la négociation $[\ldots]^{7} \gg$ Ces deux intervenants, ne définissent pas clairement, à notre avis, la notion de vente et de négociation.

\section{Les organismes de formation}

Pour des raisons de déontologie, nous ne donnerons pas le nom des organismes consultés $^{8}$, mais nous constatons la confusion des genres y compris dans le titre de certains séminaires proposés.

- Organisme A. Titre d'une formation:

«Séminaire de vente et de négociation », avec dans le programme, les deux points particuliers suivants : conduire une négociation étape par étape, argumenter, traiter les objections et conclure.

- Organisme B. Cet organisme propose des séminaires spécialisés par types de clientèles; par exemple: "Vendeurs, déjouez les pièges des acheteurs » et pour les acheteurs: «La négociation d'achat ». Comme s'il n'y avait pas matière pour les acheteurs à " déjouer les pièges des vendeurs ${ }^{9}$ » Ce même organisme propose, entre autres, dans ses programmes: négocier en $\mathrm{B}$ to $\mathrm{B}$, la vente de solutions, maîtriser la vente, la négociation commerciale, savoir vendre le prix et les conditions, la négociation commerciale par téléphone, le perfectionnement à la vente pour vendeurs, sachez vendre votre valeur ajoutée. Pour un salarié cherchant une formation, comment choisir entre la notion de vente et celle de négociation?

- Organisme C. Cet organisme propose son séminaire «phare » qui s'intitule: « vendre et défendre vos prix »

Dans le descriptif de ce séminaire, on peut lire: «Cette conférence intensive fera découvrir à vos commerciaux la façon de sortir des pièges de l'acheteur chevronné et d'éviter de donner des remises injustifiées; elle leur apprendra à présenter un prix, à le vendre, à négocier habilement et à amener un client à accepter vos conditions. » Le plan du séminaire est encore plus explicite: surmonter sa résistance au prix, maîtriser la vente du prix, résister aux clients qui marchandent, négocier au plus juste.

Quelle est la place de la négociation dans la vente ou l'inverse? Ces programmes ne sont pas, à notre avis, suffisamment explicites. En outre, ces trois organismes ont en commun de mettre en «négatif » le rôle des acheteurs et de dénier à ces mêmes acheteurs d'avoir, eux aussi, à vendre et négocier des conditions, en particulier de prix, auprès de leurs fournisseurs.

\section{Les enquêtes}

Nous avons, ces trois dernières années, menés et fait mener des enquêtes sur le thème général «Quelle différence faitesvous entre la vente et la négociation ${ }^{10} »$. La plupart des enquêtes menées ne sont pas

7. Action Commerciale, $\mathrm{n}^{\circ} 204$.

8. L'auteur tient à la disposition des lecteurs les preuves écrites de ce qui est affirmé ici; tous ces organismes sont très connus (et réputés) sur le marché français de la formation.

9. On retrouve dans l'organisme A cité plus haut, la même approche pour le stage: « Déjouer les stratagèmes des acheteurs » avec l'absence notoire d'un séminaire du style: « Déjouer les stratagèmes des vendeurs »...

10. Plusieurs de ces enquêtes ont été menées par les étudiants de l'ISGI, master dépendant de l'ESC de Lille; elles ont été réalisées avec l'appui et sous le contrôle du professeur F. Philippon. L'auteur tient à remercier chaleureusement les acteurs de ces travaux. 
scientifiquement parlant, valables, bien souvent parce que l'échantillon retenu est trop faible. Notre objectif n'était pas de faire un travail statistiquement irréprochable, mais uniquement de vérifier sur le terrain le manque de clarté entre les deux notions, vente et négociation.

$1^{\mathrm{e}}$ enquête. Cette étude a pris, comme public interrogé, un petit échantillon (20 personnes) mais très exhaustif puisqu'il allait d'un restaurateur à un avocat, d'un chef d'entreprise à un chauffeur de taxi. La question de fond posée était: « Faites-vous une différence entre la vente et la négociation personnelle et professionnelle? » Les résultats ne sont pas discriminants.

$2^{\mathrm{e}}$ enquête. Dans ce cas, la confusion a été augmentée par l'apport comparatif d'une notion complémentaire, à savoir la médiation. Les résultats ne sont pas concluants; les personnes interrogées connaissent bien les termes mais sont incapables d'expliciter clairement chaque notion. $3^{\mathrm{e}}$ enquête. Celle-ci a été un peu plus loin que les deux premières puisqu'elle distingue dans un premier temps les différences entre vente et négociation et dans un deuxième temps, en identifie les points communs. À notre avis, la méthode utilisée et les items mis en évidence ne sont pas inintéressants mais n'apportent pas de réponse claire à notre question de fond.

Quelques exemples sont présentés dans le tableau 1.

$4^{\mathrm{e}}$ enquête. Cette enquête est peut-être celle qui se rapproche le plus de notre problématique. Ci-après, quelques résultats.

1. Faites-vous une différence entre vente et négociation?

Réponse: oui : $82 \%$; non: $18 \%$

2. Un négociateur est-il forcément un bon vendeur?

Réponse: oui : $12 \%$; non: $88 \%$

Pour ces deux premières questions, les personnes interrogées caractérisent la différence par ces trois critères: le rapport de

\begin{tabular}{|l|l|l|}
\hline \multicolumn{2}{|c|}{ Tableau 1 } \\
\hline $\begin{array}{c}\text { Paractères particuliers } \\
\text { à la vente }\end{array}$ & \multicolumn{1}{c|}{$\begin{array}{c}\text { Points } \\
\text { communs }\end{array}$} & $\begin{array}{c}\text { Caractères particuliers } \\
\text { à la négociation }\end{array}$ \\
\hline Pas de conflit & Terrain d'entente & Née d'un conflit \\
\hline $\begin{array}{l}\text { Contact physique non } \\
\text { nécessaire }\end{array}$ & Communication & Contact physique indispensable \\
\hline Vente sans vendeur possible & Rapport de forces & $\begin{array}{l}\text { Au moins deux acteurs } \\
\text { indispensables }\end{array}$ \\
\hline $\begin{array}{l}\text { Rapport de forces équilibré } \\
\text { ou non équilibré }\end{array}$ & Connotation négative & $\begin{array}{l}\text { Lapport de forces équilibré } \\
\text { de marchandion prend la forme }\end{array}$ \\
\hline Vendeur souvent mal vu & \multicolumn{2}{|c|}{} \\
\hline
\end{tabular}


forces, la rentabilité économique et l'état d'esprit.

3. Pour être un bon vendeur, faut-il forcément savoir négocier?

Réponse: oui : $53 \%$; non: $47 \%$

4. Menez-vous, personnellement des actions de vente?

Réponse: oui : $53 \%$; non : $47 \%$

5. Menez-vous, personnellement, des actions de négociation?

Réponse: oui : $53 \%$; non : $47 \%$

6 . Vous arrive-t-il de transformer une négociation en vente ou inversement?

Réponse: oui : $58 \%$; non: $42 \%$

Une deuxième partie de l'enquête demandait aux sondés de donner les qualités nécessaires pour être un bon vendeur et un bon négociateur. Voici le résultat:

- bon vendeur: être persuasif, être à l'écoute, avoir un bon relationnel ;

- bon négociateur: être à l'écoute, être persuasif, être patient.

Bien que ces résultats donnent de bonnes pistes de recherche, il serait vain, à notre avis, d'y trouver des résultats explicatifs à notre hypothèse de départ. Nous devons humblement reconnaître que l'ensemble de ces enquêtes, bien que sérieusement menées, ne nous a pas apporté les résultats escomptés, si ce n'est la confirmation qu'il y a une confusion faite par les professionnels comme par les néophytes (aux métiers commerciaux) entre la notion de vente et celle de négociation.

Nous avons donc procédé, dans un troisième temps, à des analyses individualisées, d'une part, grâce à des interviews directes et d'autre part, à des témoignages relatés dans la presse spécialisée. Nous livrons ces entretiens sans en faire une ana- lyse de fond, encore moins une synthèse; ces résultats « bruts » nous serviront, dans la deuxième partie, à ébaucher des pistes de solutions.

\section{Les interviews}

\section{Entretiens directs}

Entretien $1^{11}$ : il s'agit d'une équipe commerciale de 10 personnes, y compris le directeur commercial. Voici les résultats :

- vendre, c'est avoir un produit que l'on vend, même si la personne n'en a pas besoin;

- négocier, c'est vendre un besoin;

- le vendeur est passif, il attend le client, le négociateur va au-devant du client;

- la négociation fait partie de la vente;

- on négocie avant la vente;

- dans la vente, on échange de l'argent contre quelque chose;

- on négocie quand on n'est pas obligé de conclure une vente ou un achat;

- vendre, c'est conclure, c'est l'étape finale;

- vendre, c'est lorsque les conditions sont fixées; négocier, c'est déterminer ces conditions;

- négocier se fait avant une vente;

- il peut y avoir vente sans négociation;

- vendre, c'est convaincre;

- vendre est toujours lié à de l'argent;

- c'est un peu le problème de la poule et de l'œuf!

Entretien 2: «Pour vendre ses produits, l'entreprise est confrontée à plusieurs étapes, parmi lesquelles se trouve la négociation. C'est une étape essentielle de la vente où l'on parle avec les acheteurs de conditions de vente, de prix, de la quantité

11. Société ILD, imprimerie de 160 personnes située à Ruitz. 
[...]. La vente est une résultante de la négociation. $^{12}$ »

Entretien 3: «La vente et la négociation sont des notions différentes, mais, en même temps, ce sont des notions semblables. La vente est une conséquence de la négociation. La bonne négociation mène à la bonne vente. ${ }^{13}$ »

Entretien 4: «La négociation est une phase de la vente lors de laquelle une relation gagnant/gagnant est recherchée [...] Là où la vente et le profit sont les maîtres mots, la négociation est parfaitement intégrée et nécessaire à la réussite de la vente. ${ }^{14}$ » « La négociation est la phase préalable à l'acte de vente du point de vue commercial. Il peut y avoir vente avec ou sans négociation, comme il peut y avoir négociation avec ou sans vente. Autrement dit, les deux ne sont ni incompatibles ni aimantés (l'un n'entraînant pas forcément l'autre). La vente est l'acte final, la signature du contrat; elle constitue donc une fin en soi. »

\section{Témoignages}

1. « Les seuls critères déterminants (pour recruter nos commerciaux, ndlr) sont ceux qui relèvent de la compétence et du comportement [...] Il faut avoir non seulement de la motivation, mais également une aptitude à la négociation et une orientation client. ${ }^{15}$ »

2. « Nous attendons de nos commerciaux d'acquérir cette expertise, d'avoir une expé- rience dans la vente aux professionnels, de savoir négocier et de savoir conduire une relation de partenariat global avec nos clients. ${ }^{16}$ »

3. « Négocier? Nos vendeurs n'ont pas le choix. Avec la généralisation de la reprise des véhicules, les clients jouent tour à tour à l'acheteur puis au vendeur. ${ }^{17}$ »

4. «Les meilleurs négociateurs sont ceux qui dénoncent, le lendemain de la signature, un contrat ratifié la veille. ${ }^{18}{ }$

Les interviews et témoignages reproduits plus haut confirment bien le sentiment de confusion entre négociation et vente; il est à noter que la plupart des personnes questionnées exercent des fonctions commerciales; en tous cas, ils ne semblent avoir compris, à notre avis, les questions qu'au travers du prisme des affaires.

\section{La littérature spécialisée}

La littérature sur la vente et la négociation est tellement pléthorique, que nous ne citerons que quelques exemples pour expliciter notre propos.

Hassan Souni, donne déjà le ton dans le titre de son ouvrage: Savoir négocier pour mieux vendre. Dans son introduction, il précise: « Il faut être un véritable homme de métier, c'està-dire un négociateur professionnel. Y a-t-il donc une telle différence entre un vendeur et un négociateur commercial? Oui, la même qu'entre un apprenti et un maître, ou entre un technicien et un ingénieur ${ }^{19}$ ».

12. Terliklaeva Zhupar, directrice commerciale de la société Kazahoil (Kazakhstan).

13. Kislitsina Nahalya, directrice financière de la société Cadeco.

14. Les deux personnes suivantes ont souhaité garder l'anonymat; l'une est commerciale dans les systèmes informatiques, l'autre dans la téléphonie mobile.

15. Interview de Florence Berthier, société Coca-Cola, Le Figaro Entreprise, 10 juin 2002.

16. Interview de Bernard Cohen-Solal, société ASAP Sofware, Le Figaro Entreprise, 10 juin 2002.

17. Interview de Guy Gimenez, chef des ventes Renault, L'Entreprise, ${ }^{\circ} 203$.

18. X, professeur de négociation dans une grande école de commerce; il va de soi que nous nous opposons formellement à ce type d'affirmation!

19. Éditions Demos, 1998, p. 10. 
Anne Macquin affirme: «L'activité de vente, dont on ne peut que saluer la revalorisation, est souvent appréhendée de manière restrictive. Elle est réduite aux seules opérations de négociation [...] ». L'auteur conclut, dans la présentation de son ouvrage: "Cet ouvrage s'articulera autour de ces trois niveaux de la vente, en distinguant organisation des moyens, gestion des moyens et négociation. ${ }^{20}$ »

Guy Charon et Laurent Hermel débutent leur introduction par: "Chacun de nous, dans sa vie personnelle comme dans son univers professionnel, est en permanence en situation de négociation. ${ }^{21} »$, alors que leur ouvrage s'intitule: La Vente, la nouvelle donne des forces de vente.

Patrick Roussel, dans son chapitre « Vers une vente gagnant/gagnant » explicite les trois étapes du vendeur, dont la dernière: « C'est le concept du vendeur conseil et de négociation gagnant/gagnant. ${ }^{22}$ »

Bernard Balmelle, dont l'ouvrage s'intitule La Vente, écrit: "Une négociation commerciale a pour but d'amener un client ou un prospect, par une technique d'entretien appropriée, à adhérer à une proposition inhabituelle. ${ }^{23}$ »

Citons, pour terminer cette section, un ouvrage où notre problématique se retrouve dans le titre: Négocier pour vendre ${ }^{24}$. Nous n'avons pas trouvé non plus, dans la littérature spécialisée, une réponse cohérente à la problématique de départ mais grâce à l'ensemble des résultats de cette première par- tie, nous allons tenter de formuler, dans la deuxième partie de cet article, des propositions explicatives.

\section{II. - PROPOSITIONS}

Dans cette deuxième partie, nous allons essayer de comprendre pourquoi la vente et la négociation ne sont pas perçues de manière dichotomique par l'ensemble des acteurs décrits dans la première partie de cet article et, par extrapolation, à l'ensemble des personnes concernées par les métiers de la négociation. Nous en formulerons des propositions. Pour la petite histoire, il est étonnant de constater que ce phénomène est relativement nouveau; il $\mathrm{y}$ a plus de 20 ans, la notion de négociation commerciale n'existait pratiquement pas sur le marché, y compris aux États-Unis. Pour preuve, la revue Harvard-l'Expansion a consacré un volume en 1981 sur la vente; c'est d'ailleurs le titre de l'ouvrage. Pas une seule fois, sur les 153 pages et les différents articles (français, nord-américains et britanniques), le mot «négociation » n'apparaît. Nous voyons, à partir de ces constatations, trois grands critères explicatifs: la mauvaise image de marque, surtout dans les pays latins et en particulier en France, de la vente commerciale; l'évolution du métier de vendeur; la confusion faite entre « vente » et « vente commerciale » et entre « négociation » et « négociation commerciale ».

20. Vente et Négociation, Précis Dalloz, 1993, p. 3.

21. La Vente, édition Economica, 1997, p. 27.

22. Les règles d'or de la négociation commerciale, Mémento Foucher, 2002, p. 7.

23. Chotard et associés éditeurs, 1989, p. 84.

24. de Martin et Lepage (2002). 


\section{Image de la vente commerciale}

L'image, plutôt négative, de la vente commerciale est illustrée par les témoignages suivants :

- L'APM ${ }^{25}$ a consacré une de ses revues trimestrielles au thème : "Le Dirigeant et la Vente ». Dans le chapitre «Les enjeux de la vente ${ }^{26}$ », l'auteur précise: «Pour les membres APM, le terme de vente est porteur d'ambiguïtés ; l'expression a parfois le don d'énerver. Certains adhérents ressentent même un malaise à s'exprimer à son propos; « $\mathrm{Ne}$ dites pas à ma mère que mon métier est de vendre, elle me croit dirigeant d'entreprise ». D'autres occultent facilement le sujet: "Je ne connais rien à la vente de l'entreprise car je suis ingénieur, chef d'unité de production $\gg[\ldots]$ L'auteur termine ce préambule par un avis contraire: " D'autres adhérents souhaitent appeler un chat un chat et brusquent leurs homologues en leur rappelant que la finalité de toute entreprise est de rencontrer un client et d'échanger avec lui plus de choses que de l'argent. »

- Dans un ouvrage collectif consacré à la formation des dirigeants, Anne Macquin ${ }^{27}$ souligne: « La vente est un domaine où foisonnent encore les idées reçues, les recettes, les stéréotypes et les $a$ priori négatifs; qui n'a pas entendu de propos tels que: «La vente, c'est inné, ça ne s'enseigne pas ». « Moi, j'ai une recette infaillible pour recruter de commerciaux, je considère leur stabilité dans leur vie professionnelle et affective ». « La vente, c'est bien, à condition d'y rester le moins possible. » Cet archaïsme qui caractérise la vente en fait un domaine ouvert aux pratiques magiques. Nombreux sont les gourous et autres voyants extralucides qui proposent de mettre leurs dons au service d'objectifs divers: reconquérir l'être cher, retrouver un objet perdu, guérir d'une maladie... Lesquels incluent une réussite dans la vente! À l'opposé, la recherche est le domaine du rationnel.

- Dans un article intitulé : « La vente bientôt rebaptisée ${ }^{28} »$ Francis Schillio de l'ICS de Strasbourg, auteur d'une thèse de doctorat sur "Image de la vente et motivation pour les carrières commerciales » affirme: «La vente n'attire pas particulièrement les étudiants. Problème d'image ambiguë, métier difficile, stressant, contraignant, les raisons ne manquent pas pour expliquer cette désaffection. Le mot « vente » lui-même a une connotation négative [...] L'abandon progressif du titre de directeur commercial au profit de business development manager exprime cette volonté de se défaire de la représentation négative du mot « commercial » ancré dans l'esprit de bon nombre de personnes. »

25. APM : Association pour le progrès du management; association qui regroupe à ce jour 3600 chefs d'entreprise (à grande majorité PME/PMI) répartis dans 180 clubs. Crée en 1986 par le CRC (Groupe HEC) et le CNPF de l'époque (Medef actuel). Revue, $\mathrm{n}^{\circ} 40$, décembre 2000.

26. Opus cité (p. 17); article sous la responsabilité de M. Bergadaà, professeur à l'université de Genève, et directrice de l'OVSM, Observatoire de vente et de stratégie marketing.

27. B. Moingeon, Peut-on former les dirigeants?, ouvrage collectif, éditions L'Harmattan, 2003, A. Macquin, p. 217.

28. Article d'A. F. Rabaud, Action Commerciale, n ${ }^{\circ} 204$. 
Ces constatations nous amènent à formuler notre première proposition:

\section{PROPOSITION I}

Le mot « vente » a progressivement ripé et est de plus en plus remplacé par le mot « négociation » à cause de l'image plutôt négative qu'il porte, surtout dans les pays de culture latine, en particulier en France.

\section{2. Évolution du métier de vendeur}

Dans ce même article ${ }^{29}$, l'auteur précise: «Les évolutions récentes et à venir qui touchent la fonction commerciale - mondialisation et commerce électronique - constituent sans doute une autre partie de l'explication; une profonde mutation est entrain de ce dérouler sous nos yeux, caractérisée par des changements structurels d'une portée encore insoupçonnée. De nouveaux intermédiaires verront le jour [...] Le commerce va muter et, par voie de conséquence, le rôle, la représentation commerciale et l'attractivité du commercial; et peut-être aussi, les intitulés de fonction. »

A. F. Rabaud en tire les conclusions suivantes. La multiplication des nouveaux intitulés de fonctions commerciales résulte certes d'un phénomène de mode, mais pas seulement. La multiplication des réseaux (concessionnaires, revendeurs) et l'apparition récente du e-business entrâ̂nent une réorganisation profonde de la direction commerciale et génèrent l'apparition et la création de nouveaux postes.
Nous allons compléter cette approche sur l'évolution du métier de vendeur en conservant la « confusion » décrite dans la première partie de cet article. L'évolution du métier de négociateur d'affaires s'explique par plusieurs facteurs ; complexification croissante du marché et concurrence notamment internationale plus intense; meilleure connaissance des offres concurrentes par le vendeur et de la structure des coûts par les acheteurs; développement d'approches stratégiques, aussi bien pour le vendeur que pour l'acheteur; développement de nouvelles formes de distribution par des réseaux de type vente par correspondance, marketing direct, télé-achat, etc. Ces évolutions affectent aussi bien les métiers d'acheteurs que de vendeurs [...] Il faut, en outre, tenir compte du fait que si dans les grandes entreprises les fonctions sont bien identifiées, il n'en est pas de même dans les moyennes et petites entreprises, où le négociateur, très souvent le chef d'entreprise, se trouve alternativement dans la position d'acheteur ou de vendeur ${ }^{30}$.

À partir de ces affirmations, nous pouvons formuler notre deuxième proposition:

\section{PROPOSITION II}

Le métier de vendeur (et celui d'acheteur) s'est transformé en métier de négociateur en raison de l'évolution de la complexité de la distribution commerciale, qu'elle soit locale, nationale ou internationale. 


\section{Confusions}

Nous entendons, par confusion le mélange des genres, de type: «La négociation commence quand la vente a échoué 31 » à l'affirmation: "Le processus de vente créé un besoin (une envie) et pour le satisfaire, il faut utiliser des techniques de vente qui sont suffisantes dans de nombreuses situations. Dans le cas contraire, il faut rentrer en négociation. ${ }^{32}$ » Ou bien: « La négociation commence quand le vendeur ne peut plus rien donner. ${ }^{33} »$ Un autre intervenant affirme: «Il faut faire évoluer les populations commerciales vers la négociation pour ceux qui en ont le talent ${ }^{34}$. » Nous allons faire face à la première confusion qui concerne la vente en elle-même.

\section{La Vente}

La vente regroupe, en réalité, deux grands domaines :

- la vente commerciale proprement dite, où le vendeur va conclure un contrat de fournitures et/ou de services contre une rémunération fournie par l'acheteur ;

- la vente d'idées à base de conviction et d'influence, sans avoir nécessairement de retour monétaire ou de contrat.

Exemple 1: «À Matignon, on considère que parler financement - la suppression d'un jour férié étant particulièrement dure à vendre - avant de savoir précisément à quoi on va l'affecter, est une erreur, une façon de mettre la charrue avant les bœufs, en somme ${ }^{35}$. »

Exemple 2: «L'acheteur a un travail de vendeur, car il doit vendre à l'intérieur de son entreprise les propositions qu'il a reçues et choisies. ${ }^{36}$ »

L'ensemble de ces constatations nous permet de formuler notre troisième proposition.

\section{PROPOSITION III}

Il y a deux grands types de vente:

- la vente commerciale, qui se traduit par un accord officialisé par contrat à base d'un échange monétaire ;

- la vente d'idées qui se traduit ou non par un accord entre les parties, en général sans contrat et sans contrepartie monétaire.

\section{La négociation}

La négociation couvre un champ très large d'activités: négociation commerciale ou d'affaires, négociation sociale (entre partenaires sociaux), négociation internationale (avec la problématique interculturelle), négociation diplomatique, négociation interne (hiérarchique et organisationnelle). On constate néanmoins, et la première partie de cet article le démontre amplement, que le mot négociation évoque en premier lieu la négociation commerciale (avec des

31. Mémoire de fin de $3^{\mathrm{e}}$ année, option vente, ESC de Lille, 1998.

32. P. Korda de la CEGOS, lors de la Conférence sur l'évolution des métiers de commerciaux, Salon de la formation continue, Paris, 20 mars 2001.

33. P. Korda, idem.

34. R. Beraha de COREF Développement, lors de la Conférence sur l'évolution des métiers de commerciaux, Salon de la formation continue, Paris, 20 mars 2001.

35. Les carnets de C. Pegard, Le Point, $\mathrm{n}^{\circ} 1624$ du 31 octobre 2003.

36. P. Rabut de la société AIF, lors de la Conférence sur l'évolution des métiers de commerciaux, Salon de la formation continue, Paris, 20 mars 2001. 
aspects souvent négatifs, du type: «le troc ») et le métier de négociateur celui de négociateur commercial (par exemple, les agences immobilières, en France, n'emploient pas de vendeurs mais des négociateurs). Nous pouvons, à partir de ces constatations, formuler notre quatrième proposition.

\section{PROPOSITION IV}

Lorsque le mot « négociation » est employé, il est souvent perçu comme processus pour régler des affaires, sans que le substantif « commercial » n'apparaisse nécessairement.

Il en est de même pour le métier de négociateur.

\section{Vente et négociation}

Pour formuler notre cinquième proposition, nous partirons des définitions suivantes:

C. Dupont et P. Audebert ${ }^{37}$ affirment:

«Bien qu'une distinction soit parfois difficile à faire, on peut cependant soutenir que la négociation ne doit pas être confondue avec les techniques de vente. Certes, ces deux activités ont comme point commun la recherche d'un accord, c'est-à-dire, en définitive, une prise de commande ou un contrat. Une des caractéristiques fondamentales des techniques de vente est qu'elles s'appuient sur l'importance de l'argumentation et sur les aspects comportementaux, envisagés souvent de manière unilatérale. Dans la négociation, les protagonistes se centrent beaucoup plus sur les interdépendances, sur la dimension stratégique et sur la recherche commune d'une solution (ce qui dépasse la simple commande) et sur une différenciation plus fine des techniques et des tactiques. Il est d'ailleurs regrettable que trop souvent on associe négociation à marchandage alors que le processus est beaucoup plus large; s'il apparaît difficile de négocier sans vendre, c'est-à-dire sans convaincre et obtenir l'adhésion de l'autre, on peut parfaitement vendre sans négocier. »

Ces lignes ont été écrites en pensant aux ventes et aux négociations commerciales, mais elles s'appliquent parfaitement à la vente d'idées et/ou aux négociations autres que commerciales. Les exemples ci-après en sont l'illustration.

\section{Exemple 1}

«Deux ans après le vote sur la chasse, la colère de Charles de Courson (député d'opposition à l'époque, ndlr.) n'est pas retombée. En séance, il a déposé 50 amendements contre ce texte « inapplicable »; et, hors de l'hémicycle, au sein du groupe d'études sur la chasse (les chasseurs de l'Assemblée), il a réussi à « en faire tomber » un certain nombre, notamment celui sur l'élection des présidents de fédération. J'ai lancé aux Socialistes qu'ils s'arc-boutaient sur un système mis en place par Vichy. Ca les a fait craquer, dit-il, pas peu fier d'avoir au passage décroché le soutien des communistes. ${ }^{38}$ »

Dans ce cas, c'est bien l'argumentation et l'influence, autrement dit la vente d'idées, qui a servi à obtenir un accord. ${ }^{39}$

37. Opus cité, p. 60.

38. Article dans L'Entreprise, $\mathrm{n}^{\circ} 203$, septembre 2002.

39. L'avenir nous dira, ou pas, s'il y a eu des contreparties secrètes données par C. de Courson; dans ce cas, il y aurait également négociation. 


\section{Exemple 2}

« À la suite de la visite discrète du ministre mexicain des Relations extérieures, Jorge Castañeda, à Colin Powell, le Mexique se serait vu proposer un marché: l'annulation de la dette contractée envers les États-Unis pour ses achats d'eau contre un vote favorable au Conseil de sécurité en faveur d'une intervention armée en Irak. » ${ }^{40}$

Ici, le jeu des contreparties paraît primordial par rapport à l'argumentation, il y a donc beaucoup plus négociation que vente. A. Macquin ${ }^{41}$ explicite de manière très élégante la différence entre vente (commerciale) et négociation: « Le rôle du vendeur est de faire émerger les besoins du client ou prospect et de montrer en quoi le produit proposé y répond. On s'éloigne de l'image d'Épinal du commercial hâbleur et baratineur. Le bon vendeur est celui qui sait amener sont interlocuteur à exprimer ses besoins, centrer le débat sur ceux auxquels son produit peut répondre et mettre en face d'eux des bénéfices consommateurs, c'està-dire des caractéristiques produit a priori neutres, exprimés en tant que réponse à des besoins. » Pour définir la négociation, cet auteur part du Petit Robert: "Série d'entretiens, d'échange de vue, de démarches qu'on entreprend pour parvenir à un accord, pour conclure une affaire ». Et poursuit: "La négociation ainsi définie englobe les interactions de vente, mais va au-delà pour inclure toutes les interactions où des individus ayant des intérêts divergents essaient de dégager un accord en fai- sant usage de la parole uniquement. » Nous pouvons illustrer les propos d'A. Macquin par l'exemple suivant: "Quelles clauses confidentielles accompagnent la commande passée par EasyJet de 120 Airbus A 319? [...] Les négociations, entre Airbus et EasyJet n'auraient pas porté uniquement sur le prix des appareils. EasyJet aurait aussi cherché à obtenir des créneaux horaires sur Orly pour concurrencer Air France. ${ }^{42}$ » Où la négociation rejoint la vente. Nous constatons, grâce à ces exemples, que la vente, qu'elle soit commerciale ou d'idées, est basée avant tout sur la rhétorique, la persuasion et l'influence formalisés par Aristote il y a plus de 25 siècles! Ces méthodes explicitent l'art de construire un discours (élaborer sa pensée) et l'art de persuader et de convaincre (c'est l'art de la preuve ${ }^{43}$. Quant à la négociation, si la rhétorique y est aussi très importante, elle est basée avant tout sur l'échange, c'est-à-dire le jeu des concessions et contre-concessions, autrement dit, l'octroi mutuel de contreparties pour arriver à un accord. Nous formulerons notre cinquième et dernière proposition sous une double forme:

\section{PROPOSITION V (A)}

- Vendre c'est convaincre, influencer.

- Négocier, c'est échanger.

- On peut vendre sans négocier.

- On ne peut pas négocier sans vendre.

40. Article publié dans Le Point, 25 octobre 2002.

41. Opus cité, p. 221.

42. Article de T. Vigoureux, Le Figaro Entreprises, 28 octobre 2002.

43. Un très bon ouvrage reprend toutes ces méthodes, Neirynck (2003). 
Nous terminerons par une sous-proposition qui définit l'interaction vente-négociation:

\section{PROPOSITION V (B)}

La vente agira à trois niveaux :

- Optimiser au maximum l'échange.

- Mettre en valeur l'échange (la concession).

- Faire accepter l'échange par tous les partenaires.

Il est à noter que cette double proposition s'applique aussi bien aux ventes commerciales qu'aux ventes d'idées et à toutes les formes de négociation. Nous voudrions conclure cette deuxième partie par les deux points suivants :

1. À partir des travaux de Guérin, Laborey et Angeli (1979), nous proposons une nouvelle définition:

- Vendre consiste à faire se convaincre mon interlocuteur que l'idée, le service ou le produit que je lui propose répond au mieux à ses idéaux, ses valeurs, ses intérêts (enjeux), ses besoins (matériels).

- Négocier consiste à analyser ensemble une situation où existe un minimum d'intérêts communs bien que les intérêts de chaque partie semblent opposés et que chacun cherche à « vendre » quelque chose à l'autre, à l'aide de concessions et/ou de propositions originales non prévues au départ, afin de parvenir à un accord satisfaisant de part et d'autre.

2. Nous souhaiterions tenter une synthèse de l'ensemble des travaux répertoriés dans cet article en proposant le tableau récapitulatif ci-après.

\section{CONCLUSION}

En guise de conclusion, nous aimerions soumettre le dialogue suivant qui fait référence à une situation qui peut être analysée comme une vente ou une négociation. Le

\begin{tabular}{|c|c|c|}
\hline \multicolumn{3}{|c|}{$\begin{array}{c}\text { Tableau } 2 \\
\text { INFLUENCE DES ITEMS SUR LA VENTE ET LA NÉGOCIATION }\end{array}$} \\
\hline Items & Vente & Négociation \\
\hline Art de la conviction (rhétorique/argumentation) & forte & faible \\
\hline Séduction & forte & faible \\
\hline Influence & forte & faible \\
\hline Échange (jeu des concessions) & très faible & très forte \\
\hline Créativité (Proposition de solutions originales) & très forte & forte \\
\hline Communication & forte & forte \\
\hline Confiance & moyenne & moyenne \\
\hline Incidence du rapport de forces & faible & forte \\
\hline
\end{tabular}


comédien, Michel Serrault, dans son dernier ouvrage ${ }^{44}$, raconte la négociation qu'il a menée avec le célèbre acteur Pierre Fresnay qui était, à l'époque, codirigeant du théâtre de la Michaudière avec son épouse, la non moins célèbre Yvonne Printemps.

« Je me suis retrouvé devant lui dans le bureau directorial pour signer mon contrat [...]. C'est peu dire que j'étais impressionné [...] Dans ce bureau, Pierre Fresnay semblait plus intimidé que moi! Il se racla la gorge et attaqua, de sa voix qui fit le bonheur de quelques imitateurs, mais qui n'était pas tout à fait la même sur scène et dans la vie.

- Eh bien voilà mon cher ami... Il faut bien que nous parlions un peu... Puisque vous allez jouer chez nous... Vous savez combien je vous admire.... Je suis sûr que nous allons nous entendre... Voyons... Avec Yvonne nous avons pensé... Et vous me direz bien sûr comment vous voyez ça... Si vous êtes d'accord, ou si vous croyez qu'on peut... Enfin voilà la proposition qu'Yvonne et moi avons imaginée...

Il m'avança un chiffre très convenable, mais, au comble de l'émotion, je ne répon- dis pas, me contentant d'un furtif hochement de tête en signe d'approbation. Fresnay ne le comprit pas comme ça! Et mon silence l'obligea à meubler, à poursuivre son discours!

- Évidemment, me dit-il en se raclant à nouveau la gorge, on pourrait... Je veux dire qu'avec Yvonne nous pouvons envisager une somme plus élevée...

Il me donna un nouveau chiffre, sensiblement supérieur au premier. Je restai toujours muet, mais avec un mouvement du chef tout semblable. Même méprise.

- Bien sûr, soliloqua Fresnay, bien sûr... $\mathrm{Ne}$ nous tracassons pas. Yvonne tient à vous avoir, nous pouvons faire un effort complémentaire...

Il augmenta encore. J'entendis alors sortir d'un gosier desséché (le mien) un son qui évoquait l'ouverture d'une veille armoire.

- C'est très bien Monsieur Fresnay...

Bien plus tard, Pierre Fresnay m'avoua en riant:

- Jamais de ma vie j'ai souffert comme vous m'avez fait souffrir le jour de votre contrat! Faire monter les enchères sans dire un mot, je n'avais jamais vu ça!»

\section{BibliographIE}

Audebert-Lasrochas P., La Négociation, Éditions d'Organisation, 2002.

Balmelle B., La Vente, Chotard et Associés, 1989.

Charon G. et Hermel L., La Vente, Economica, 1997.

Dupont C., La Négociation, Dalloz, 1994.

Dupont C. et Audebert P., La Négociation: Applications et Exercices, Dalloz, 1994.

Macquin A., Vente et Négociation, Précis Dalloz, 1993.

Martin M.-A. et Lepage O., Négocier pour Vendre, Le Génie des Glaciers, 2002.

44. Serrault et Massot (2001, p. 211 et suiv.). 
Moingeon B., Peut-on former les Dirigeants, l'Harmattan, 2003.

Neirynck D., Tout Savoir sur la Communication Orale, Éditions d'Organisation, 2003.

Roussel P., Les Règles d'or de la Négociation commerciale, Mémento Foucher, 2002.

Serrault M. et Massot F., Vous avez dit Serrault?, 2001.

Souni H., Savoir Négocier pour mieux Vendre, Demos, 1998. 